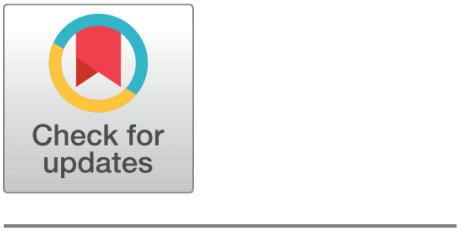

OPEN ACCESS

Received: 11.09.2021

Accepted: 11.12 .2021

Published: 21.01.2022

Citation: Satyanarayana AVS, Rao MJ, Reddy BS, Chandra Mouli K, Satya Guru TVSPV (2022) EPMA and PIXE Analyses of High Grade Metamorphic Rocks. Indian Journal of Science and Technology 15(1):

1-8. https://doi.org/

10.17485/IJST/v15i1.1651

* Corresponding author.

savs.viit@gmail.com

Funding: None

Competing Interests: None

Copyright: (c) 2022 Satyanarayana et al. This is an open access article distributed under the terms of the Creative Commons Attribution License, which permits unrestricted use, distribution, and reproduction in any medium, provided the original author and source are credited.

Published By Indian Society for Education and Environment (iSee)

ISSN

Print: 0974-6846

Electronic: 0974-5645

\section{EPMA and PIXE Analyses of High Grade Metamorphic Rocks}

\author{
A V S Satyanarayana ${ }^{1 *}$, M Jagannadha Rao ${ }^{2}$, B Seetharami Reddy ${ }^{3}$, \\ K Chandra Mouli ${ }^{1}$, T V S P V Satya Guru ${ }^{4}$ \\ 1 Department of Engineering Physics, Andhra University, AP, Visakhapatnam, India \\ 2 Department of Geology, Andhra University, AP, Visakhapatnam, India \\ 3 Department of Nuclear Physics, Andhra University, AP, Visakhapatnam, India \\ 4 Department of Chemistry, Vignan's Institute of Information Technology, AP, 530046, \\ Visakhapatnam, India
}

\section{Abstract}

Background/Objectives: The demand for exact quantification analyses in complex ore assemblages is increasing rapidly as mineral processing studies become more sophisticated. In response to this need, an Electron Micro Probe Analysis and Proton Induced X-ray Emission complementary based analytical tool procedure for the chemical characterization of minerals was employed to study the metamorphic rocks. Methods : In this work, we compare the quantitative analysis of high-grade metamorphic rocks by proton induced X-ray emission (PIXE) with that of the previous analyses by electron probe microanalysis (EPMA). During this investigation, the PIXE analytical method on a nuclear microprobe was added to the previously well-known EPMA for the chemical quantification of high-grade metamorphic rocks from the Charnockite Hill, Visakhapatnam, A.P., India. For the evolution and geochemical characterization of the metamorphic rocks, the above two methods are compared and discussions are made with respect to certain elements. Findings: Particle-induced $\mathrm{X}$-ray emission is well-matched except for certain elements for the chemical characterization of metamorphic rocks belonging to earth material samples. This comparison with EPMA demonstrates the elemental quantification characteristics for such analyses. Not only is an exploratory study of proton induced X-ray emission benefit presented, but also a synopsis of comparison with electron probe microanalysis aimed at a responsible reply to metamorphic rock analytical questions. Novelty: The complementary technique, especially EPMA, is required to fill the elemental gaps that are not determined in the case of matrix complex charnockite compositional analysis by PIXE with $3 \mathrm{MeV}$ and a Si (Li) detector.

Keywords: Electron and Proton Microprobes; Complementary Techniques; Metamorphic Rocks; Elemental Analysis 


\section{Introduction}

The charnockites of Visakhapatnam, A.P. and India's most prominent airport, a 136' hill Figure 1, are being extensively quarried due to the airport extension. In the geochemical study of these metamorphic rocks Figure 2 from the Eastern Ghats, Visakhapatnam, AP, India, samples with halogen-rich minerals have been identified. Biotite, hastingsite, and apatite are halogencontaining minerals in charnockite composition, and this report deals primarily with their occurrence and composition. One of the coarse-grained garnet-biotite-hypersthene granodioritic charnockites ${ }^{(1)}$ occasionally contains allanite and is believed to be of palingenetic origin. Biotite is a common mineral in the palingenetic charnockites. Hornblende and apatite occur in minor amounts in these rocks. The rock sample containing halogen-bearing minerals belongs to the younger granodiorite charnockitic family.

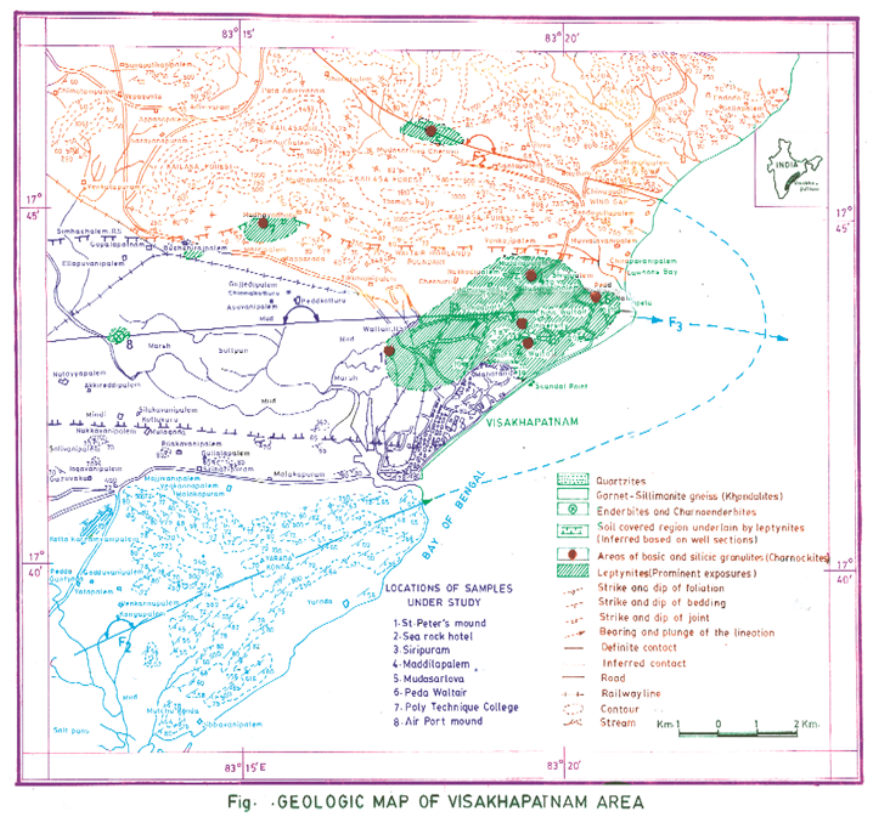

Fig 1. Visakhapatnam airport area, the central portion of a charnockite hill After Sriramadas and Rao (1979)

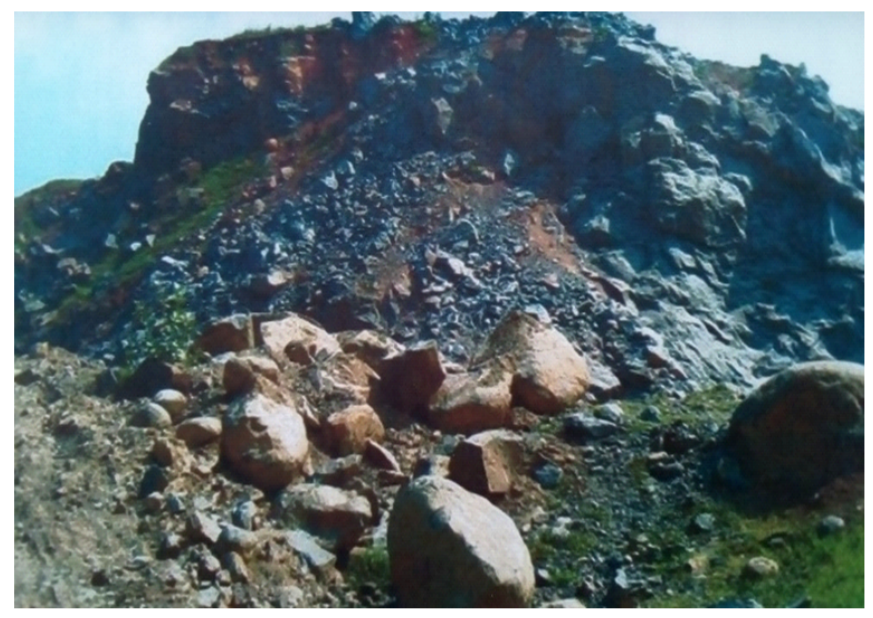

Fig 2. Chatrnockite hill of airport area, Visakhapatnam

Different generations of charnockites are present in the Eastern Ghats Belt, but the charnockitisation of granitic gneisses is yet to be documented. Charnockitic rocks that suffered granule facies metamorphism and attendant migmatization in the coastal 
India area are shown to be relicts of older ${ }^{(2)}$. The matrix high-grade metamorphic rock sample came from the terrestrial planet system, and its sample preparation for the experiment is significant in studies of metamorphic rocks and their quantification. Contaminants occurring during specimen preparation are part of the careful threat for geochemical characterization of earth materials, as are contaminants occurring during specimen preparation in the earth's solar system.

The demand for exact quantification analyses in complex ore assemblages is increasing rapidly as mineral processing studies become more sophisticated. In response to this need, an EPMA-based analytical tool procedure for the chemical characterization of minerals was developed. The analytical tool uses an electron microprobe to collect combined X-ray maps. EPMA and X-ray element distribution maps of grains are acquired in parallel, enabling direct comparison between elemental concentrations and textural features. An automated cluster recognition technique has been developed for systems characterised by highly complex mineral phase assemblages ${ }^{(3)}$. Particle-induced X-ray emission ${ }^{(4)}$ is well suited for the chemical characterization of metamorphic rocks belonging to earth material samples. Not only is an exploratory study of proton induced X-ray emission benefit presented, but also a synopsis of comparison with electron probe microanalysis aimed at a responsible reply to metamorphic rock questions.

\subsection{Microanalysis Using an Electron Probe}

EPMA experimented with a projectile of electrons (energy range; 5 to $30 \mathrm{keV}$ ) by interacting with a small volume of a target and gathering the $\mathrm{X}$-ray photons thereby induced by the various elemental specimens. The characteristic X-rays emitted by the target composition can be easily quantified by gathering through Wavelength Dispersive Spectroscopy spectra. An electron projectile with sufficient energy is allowed to crash into the incident to liberate energy from the target. These electron-target interactions liberate heat, but they also yield both X-ray and derivative electrons. An X-ray is emitted by inelastic collisions of the bombarding electrons with lower shell electrons of atoms in the target. When a lower-shell electron is vacated from its orbit, leaving a vacancy, a higher-shell electron fills this vacancy and must shed some energy as an X-ray to do so. This quantified $\mathrm{X}$-ray is characteristic of the element in the sample. EPMA ${ }^{(5)}$ is a fully quantitative and qualitative tool of non-destructive multi-elemental analysis with ppm sensitivity. Over several days, common quantification to $1 \%$ weighting is accomplished. It is the most accurate, precise and all elements from boron to uranium can be analyzed.

In the electron beam case, the electron mass and target electrons are the same. However, in the case of the proton, its mass is greater than that of the target electron. Therefore, the overall kinetic energy loss is very small in the proton projectile case, and its direction of travel is almost unchanged. The momentum is smaller in the case of the electron beam than in that of the proton beam, and hence electron projectiles suffer a larger fraction of energy loss per encounter. Because of huge mass fraction differences, the bremsstrahlung is higher in the electron beam case than in that of the proton beam. Choosing the most suitable analytical tool for multi-elemental quantification of matrix metamorphic rocks is not always possible, since several experimental factors based on the target and the analytical requirements have to be considered. In this study, PIXE general information about the elemental composition of metamorphic rocks is presented, along with a comparison with EPMA.

\subsection{Microanalysis Using a Proton Probe}

H. Moseley experimented with the characteristic X-rays induced when materials were bombarded with cathode rays. Since electrons are particles too, this is the first report of particle induced X-ray emission. The first described the spectrometer and pointed out that its elemental samples were contaminated with impurities. In his second, he analytically calculated $\mathrm{K}$ and $\mathrm{L}$ line energies. PIXE using $\mathrm{Si}(\mathrm{Li})$ detectors suggested that the trace-element limit of detection could be as low as ppm and analysed geological samples.

With a systematic technique to estimating major, minor, trace element, and REE elements of rock composition, an important attempt has been made to examine the accuracy and precision of the PIXE fundamental parameters. To assist the comparison of the PIXE data, complementary electron probe micro-analysis measurements with earlier characterizations were used ${ }^{(6)}$. The $\mathrm{X}$-ray production cross-sections, not the ionisation cross-sections, must be determined with considerable accuracy for any PIXE measurement. When the particle velocity equals the average velocity of the electron in that shell, the cross-sections for the production of an inner-shell vacancy rise sharply as a function of particle energy, reaching a maximum when the particle velocity equals the average velocity of the electron in that shell. The cross-section discusses starting a slow reduction when the energy of the projectile particles is increased further.

Bremsstrahlung processes ${ }^{(7)}$ contribute to the PIXE spectrum, and their contribution is dependent on incident energy and target atomic number. Incident particle bremsstrahlung, which appears to be a major cause of background at high X-ray energies, is one of the primary processes that contribute to the background. The slowing of the projectile ion in coulomb contacts with bound atomic electrons causes the emission of bremsstrahlung photons with energies ranging from zero to the projectile 
kinetic energy in PIXE. Secondary electron bremsstrahlung is a low-energy background thought to be created primarily by secondary electrons emitted from target atoms during the collision process. The noise produced by Compton scattering of rays produced by interactions of protons with the nuclei of the sample being examined can significantly reduce the detection limit of trace elements with higher atomic numbers. Gamma-ray Compton scattering from backscattered protons reacting with the nuclei of the X-ray filter or window materials inside the detector $(\mathrm{P})$ and $(\mathrm{P} \mathrm{n})$. Due to the discharge of a non-conducting specimen charged under ion bombardment, electron bremsstrahlung occurs.

The proton beam is used as an incident source in the PIXE method because it has several merits over other ion excitements like the electron microprobe. A few advantages are: 1) a higher data accumulation and hence allows faster analysis. 2) large $\mathrm{X}$-ray production cross-sections of 3) increased overall sensitivity 4) ions that are heavier 5) as the projectile's atomic number and energy increase, so do the effects of multiple ionisations. The proton energy range of $2-5 \mathrm{MeV}$ is the optimum, because it takes into account, both the variation in the cross-section for characteristic X-rays and the various effects caused by secondary electron bremsstrahlung.

\section{Materials and Methods}

The metamorphic rock samples from the interior central part of a charnockite hill near Visakhapatnam city, the Eastern Ghats, A.P, and India were chosen for the concentration of elements. Such geological samples are more significant, and the proton microprobe method is selected for the multi-elemental analysis. Two approaches to metamorphic rock characterization based on PIXE ${ }^{(8)}$ and complementary methods ${ }^{(9)}$ like EPMA ${ }^{(10)}$ have been experimented with. The above two methods of excitation are compared from analytical points of view ${ }^{(11)}$ and for the detection of concealed mineralization. By analysing them with the electron and proton microprobe technique, major and traces of important elements present in the metamorphic rocks were found.

For PIXE, measurements of thick targets are much easier for analysis, that are usually prepared in the form of pellets from previously homogenized, dried, and grind samples. Those samples may also contain an element that serves as an internal standard. In order to determine the concentration levels in both cases, the external standard is also measured. In the case of mineral samples, the sample is powdered in an agate bowl, mixed with glycerine, and smeared on a backing film. Solid rock samples are reduced to powder with a particle size of 200 mesh or lower. After being mixed with high purity graphite in 10:3 rock-graphite ratios, the rock sample was compressed into a disc shape with a $1 \mathrm{~mm}$ thickness and a $10 \mathrm{~mm}$ diameter.The sample is dried and powdered to micro-millimeter fineness. The sample employed for $3 \mathrm{MeV}$ energy protons was equipped by scattering a few mgr of rock on 4 micrometers, Mylar film, and using a drop of $0.1 \mathrm{ml}$ of polyvinyl acetate diluted with acetone as an adhesive. Then the pellets were attached to the tape of scotch and arranged on the aluminium frame.

EPMA has been experimented with ${ }^{(10)}$ by interacting a small volume of a target with an interacting electron microprobe (typical energy range $=5-30 \mathrm{keV}$ ) and gathering the $\mathrm{X}$-ray photons thereby induced by the various elemental species. Because of the characteristic X-ray wavelengths produced by species, the sample quantification can be easily measured by recording wavelength dispersive spectroscopy. It is a complete qualitative and quantitative method of the non-destructive elemental analytical tool for small-sized volumes of materials, with sensitivity and accuracy at the level of ppm. Previously, apatite, biotite, and hastingsite grains of charnockite metamorphic rocks were analyzed.he accelerating voltage was $20-\mathrm{kV}$ with a specimen current of 10 nanoamperes measured on a kaersutite standard. The counting time was 100 seconds and the analytical raw data was gathered for background, non-resolution, and matrix effects via MAGIC ${ }^{(12)}$. Using an arrangement of ETEC-SEM ${ }^{(13)}$ with a WDS, the fluorine analyses were obtained.

The present experimental work was combined with the well-known previous electron microprobe of the same type and same location analysis technique for the characterization of a metamorphic rock from the Charnockite Hill, Visakhapatnam. The analysis is to be followed in order to know the geochemical characterization of the metamorphic rocks using nuclear microprobe techniques ${ }^{(14)}$. Present PIXE investigations are being carried out at the ion beam laboratory, Institute of Physics, Bhubaneswar, India. This aim is to quantify various elements present in the sample and their relative intensities. Using GUPIX software, the X-ray intensities of different elements are converted into the corresponding quantifications using a standardised tool involving basic parameters, pre-determined instrument constants, and input parameters. To prove the reliability of the experimental part and other factors, the PIXE spectrum was obtained with NIST certified, standard reference material. In the case of PIXE analysis ${ }^{(15)}$, the characteristic X-ray wavelengths emitted by the target composition can be easily quantified by gathering them through the $\mathrm{Si}(\mathrm{Li})$ detector (typical energy range $=2-20 \mathrm{keV})^{(16)(17)}$. 


\section{Results}

During this investigation, the aim is to characterize the matrix metamorphic minerals by using the PIXE technique at various atomic numbers of elements. The results obtained by PIXE of the matrix composition should be compared with the previous results of charnockite metamorphic rocks, which were published in various journals in the same area and location by EPMA. Based on that, various factors related to the PIXE method with the comparison of previous EPMA techniques have been discussed in the case of matrix geomaterials. The above two methods of excitation are compared from analytical points of view and for the detection of concealed mineralization.

Previously, the experimental work was carried out using electron microprobe analysis [10] different elements were identified. The presenters used the PIXE technique at the Institute of Physics' $3 \mathrm{MeV}$ particle accelerator facility in Bhubaneswar. The characteristic X-rays of atoms of geological samples of charnockite metamorphic rock concentrations of various elements containing minerals fig- 1 and 2 were detected with the $\mathrm{Si}(\mathrm{Li})$ detector. After comparison with the previous study using electron microprobe analysis, the elemental concentrations in charnockite metamorphic rocks by using the present PIXE technique for multi-elements are different from previous analyses.

The analysis is to be followed in order to determine the evolution and biochemical characterization of high-grade metamorphic rocks using the PIXE technique. First, to know the workings of experimental systems, the PIXE spectrum is recorded with NIST certified reference material standard sample concentrations. The results of the present PIXE with $3 \mathrm{MeV}$ and $\mathrm{Si}(\mathrm{Li})$ detection with a resolution of $160 \mathrm{eV}$ at $5.9 \mathrm{keV}$ for the element testing have been validated by comparing these values with those of the standard material USGS-basalt used ${ }^{(8)}$. The PIXE results obtained from the geological samples from the Eastern Ghats, A.P., India of seven places collected in this experiment from the interior of the charnockite hill, Visakhapatnam, were recorded by the $\mathrm{Si}(\mathrm{Li})$ detector. The measured values in ppm of these various elements in each rock sample were determined. These concentrations are tabulated with errors and finally verified with the previous analytical technique, EPMA ${ }^{(10)}$

\section{Discussions}

In this paper, an attempt is made to compare the geochemistry of metamorphic rock samples using PIXE and EPMA. Quantitative compositional analysis using prior electron microprobes is a strong tool for metamorphic rock research, but it requires a strict data reduction technique to accomplish accurate composition quantification. The software should have an enhanced standardisation mechanism that enables for a pseudo-background adjustment for low ppm elements in charnockite rocks without the need to measure background values. In the case of not attained findings when compared to PIXE, a background adjustment is required for an accurate and precise computation of major, minor, and trace quantifications of elements.

The quantifications in ppm of these wide ranges of elements in each specimen were quantified using the GUPIX software with $\mathrm{Si}(\mathrm{Li})$ detection, and these quantifications with errors were derived using PIXE spectrum interpretations. A comparison of analytical methods for metamorphic rocks is used to investigate the components present in rock composition as well as the missing elements between earlier EMPA results and the current PIXE spectrum of samples.

An electron microprobe can be used to obtain high precision, accurate quantitative multi-element analysis of geological materials with high-quality imaging at a higher spatial resolution for major and trace elements from $\mathrm{B}$ to $\mathrm{U}$ in general. Because many minerals contain significant amounts of $\mathrm{OH}$ groups or $\mathrm{H} 2 \mathrm{O}$, the inability to quantify hydrogen is one of the largest disadvantages. From the foregoing data, the possibility is limited to monitoring for light elements such as lithium, beryllium, and boron, especially $\mathrm{Z}<6$. Most of the elements have detection limits of $100 \mathrm{ppm}$ or greater. When looking at trace element concentrations in the ppm or even sub-ppm range, this was a limiting factor in previous EMPA routine analysis. Chemical dating of metamorphic rock is done using the Th- $\mathrm{U}-\mathrm{Pb}$ incompatible minerals.

In the case of minor and trace elements, EPMA quantitative mapping has a lot of potential to be coupled with other mapping techniques, despite the technological advancements. EPMA explains the various issues that arise in the elemental analysis of metamorphic rocks, as well as the capacity to analyse for practically all elements. Some elements must be separated that emit $\mathrm{X}$-rays with overlapping energy and wavelength peak positions. Previously, EPMA analyses were calculated using iron oxides of elements rather than cations. As a result, cation extent and mineral formulae must be pre-measured using stoichiometric criteria.

The tracing of REEs is important in metamorphic rocks. However, EPMA of REEs continues to have issues due to the complexity of their X-ray spectra. Before performing the normal correction approach, we advise using a modified version of the programme we produced for the analysis, in which an automatic correction for interfering lines and a background determination computational method are utilised. The EPMA allows one to considerably simplify REE analyses, eliminate random mistakes, enhance the correctness and speed of microprobe determinations, and lower the limit of detection of the 
elements studied in order to solve the background determination problem. Most REEs have a detection limit of over 500 parts per million when analysed in REE-bearing materials. With a probe current range of $100 \mathrm{nA}$ at a 20 -kilovolt accelerating voltage and a 50-second counting period at the analytical station, these values were most likely obtained in metamorphic rocks.

The LOD determined from the reference formula measures virtually the quantification state that could only be obtained in the case of exact computations of overlap factors of all interfering lines ${ }^{(18)}$ for elements with diverse interference of analytical lines, such as $\mathrm{Gd}, \mathrm{Tm}, \mathrm{Eu}$, and Lu. For accurate in situ determination of $\mathrm{Th}, \mathrm{U}, \mathrm{Pb}$, and other elements, a non-destructive EPMA analytical equipment can be used. Due to detector restrictions, the element F identified in the previous is exceedingly low and was not detected. In the earlier EPMA investigation, the key element oxides of $\mathrm{Na}, \mathrm{Mg}, \mathrm{Al}, \mathrm{Si}$, and $\mathrm{P}$ were examined and revealed a $100 \mathrm{ppm}$ concentration, but PIXE exhibited a $1 \mathrm{ppm}$ range, 100 times more than detection.

PIXE ${ }^{(8)}$ was used to test several factors such as accuracy, precision, and detection limits of multi-elements measurements ${ }^{(19)}$ of metamorphic rocks. The effect of a proton spot size of $3 \mathrm{MeV}$ on rock samples was investigated using a $\mathrm{Si}(\mathrm{Li})$ detector. Because of the appeal of non-destructive procedures and the capacity to do simultaneous multi-elemental determinations, accurate, precise, and sensitive PIXE atomic and nuclear analytical methods have been widely used ${ }^{(20)}$ Except for some components from the above results, the PIXE inquiry is normally extremely suitable and sensitive when $\mathrm{Z}$ is in the range of 20 to 40 , however in this study of charnockites, the elements found in this case are $\mathrm{Z}=17$ to $\mathrm{Z}=82$.

The light elements $\mathrm{Li}, \mathrm{Be}, \mathrm{F}, \mathrm{Na}, \mathrm{Al}, \mathrm{Mg}, \mathrm{Si}$, and $\mathrm{P}$ are once again examined in the PIXE with $3 \mathrm{MeV}$ experiments ${ }^{(21)}$. It has been previously discussed, but it is possible that the low energy particle PIXE is able to detect the above elements due to suitability cross-sections ${ }^{(22)}(23)$ or by placing a more suitable detector, with the potential advantages of low background radiation, good sensitivity for light elements, and lower secondary excitation in thick targets explained. Due to low energy protons, REE cannot be discovered in crucial metamorphic rock investigations, despite the fact that the few elements are present in the form of major compositions ${ }^{(23)}$. However, key elements such as $\mathrm{K}, \mathrm{Ca}, \mathrm{Fe}, \mathrm{Cl}$, and minor elements are found with adjustments in halogen-rich charnockite metamorphic rocks.

The highest numbers of elements in media Z, such as Co, Sc, Ga, Sn, Ba, and higher Z elements, such as U, Th, ${ }^{(24)}$, and $\mathrm{REE}^{(25)}$ are not identified. The X-ray production is a function of the ionisation cross-section for the distinct electron shells $\mathrm{K}$, $\mathrm{L}$, and $\mathrm{M}$ in all methods of X-ray investigation, including low-energy proton microprobes with a thick target (Energy $=2$ to 5 $\mathrm{MeV}$ ). The cross-section of low-energy protons is greater for the $\mathrm{K}$ shells of low-Z elements and the $\mathrm{L}$ shells of high-Z elements, providing sufficient fluorescence yields for measurement. Low-energy incoming protons cannot ionise K-shell electrons in high $\mathrm{Z}$ elements ( $>55)$, and quantification is dependent on the X-ray lines employed. Interference K X-rays from low Z elements can affect L X-ray spectra, making them difficult to obtain. Complexity and spectrum interference can both lead to issues and ambiguity in the explanation.

This can make X-ray emissions from elements in low concentrations difficult to see. In situ analysis with LE-PIXE microprobes may achieve a spatial resolution of about $5 \mathrm{pm}$, which is much better than what is presented and commonly available with HE-PIXE, and has a lower LOD than electron probe micro-analysis. From the foregoing results, low energyPIXE is suitable for the study of low $\mathrm{Z}$ trace elements $(\mathrm{Z}=55)$ utilising $\mathrm{K} X$-rays. We are now able to use $3 \mathrm{MeV}$ energy PIXE in a quantitative manner to charnockite research concerns, particularly in the case of major, minor, and trace elements, as a result of this experimental work. Tests to explain the $\mathrm{k}$ cross-sections at $3 \mathrm{MeV}$ proton energies for $\mathrm{Z}=17$ to $\mathrm{Z}=48$ elements would also be useful, as would experiments to clarify the $\mathrm{L}$ cross-sections for high $\mathrm{Z}$ elements like $\mathrm{Pb}$. The contract was signed.

The detection limit of middle $Z$ elements in a metamorphic sample using a proton microprobe is less than $1 \mathrm{microgram} / \mathrm{gram}$, indicating the system's sensitivity and being connected with the signal peak and background of the fluorescence spectrum measurement. Despite the greater ionisation cross-section for low Z elements, the detection limit of PIXE is reduced. This is due to a decrease in fluorescence yield, a rise in bremsstrahlung background for lighter elements, and an increase in detector window absorption. The detection limit at $3 \mathrm{MeV}$ is below $1 \mathrm{ppm}$ when $\mathrm{Z}$ is in the range of $17-82$, according to the above data. Quantitatively, the production of $\mathrm{X}$-rays by $3 \mathrm{MeV}$ protons and prior keV energy electrons is similar.

The bremsstrahlung background, which is the primary determinant of the detection limit of several elements from $\mathrm{Cl}$ to $\mathrm{Pb}$ down to $\mathrm{ppm}$, is, however, too low to make such a measurement. Because of these energies, the detection limit for middle $\mathrm{Z}$ elements derived from the above data is low. Because the detection limits of $\mathrm{K}$ and $\mathrm{L} \mathrm{X}$-ray measurements coincide at an atomic number near 50, this analysis was done for $\mathrm{K} \mathrm{X}$-ray in the geochemistry of metamorphic rock samples for elements with $\mathrm{Z}$ below 50, and for L X-ray in the geochemistry of metamorphic rock samples for elements with Z above 50.

When the beam current is $20 \mathrm{nA}$ and the measurement time is 4 minutes, the LOD for Se, which is a medium $\mathrm{Z}$ element when measured using $3 \mathrm{MeV}$ PIXE analysis, is calculated to be $0.9 \mathrm{mg} / \mathrm{kg}$. As a result, employing PIXE with $3 \mathrm{MeV}$, traceelement analysis of less than 0.0001 percent wt or $1 \mathrm{ppm}$ is possible in metamorphic rocks. This demonstrates the utility of PIXE analysis in the $3 \mathrm{MeV}$ energy regime for metamorphic rock analysis. In the $\mathrm{Z}=30-44$ region, the detection limits in the 3 $\mathrm{MeV}$ range rapidly increase as the atomic number increases. The decrease in the characteristic X-ray production cross-section 
due to coulomb deflection and binding energy effects can explain this behaviour.

The fundamental challenge with PIXE analysis of metamorphic rock targets is the extremely strong X-ray emission from $\mathrm{Ti}$ and $\mathrm{Fe}$, which tends to obscure the minuscule trace element peaks in the energy spectrum. In the case of element analysis, interference between X-ray K lines of media elements and X-ray L lines of heavy elements or between the X-ray K lines of media elements and the X-ray L lines of heavy elements is common. In the case of some metamorphic rock samples, such interferences include: $\mathrm{Co}$ and $\mathrm{Fe} ; \mathrm{Ni}$ and $\mathrm{Co} ; \mathrm{Fe}$ and $\mathrm{Mn} ; \mathrm{Cu}$ and $\mathrm{Zn} ; \mathrm{Y}$ and $\mathrm{Rb}$ of $\mathrm{K}$ alpha and $\mathrm{K}$ beta; and corrections must be performed for quantitative element analysis. Because the L X-rays of these REE have the same distinctive K X-ray energies as low Z, the PIXE at $3 \mathrm{MeV}$ is also ineffectual for REE in rock composition ${ }^{(26)}$.

As a result, high-energy PIXE ${ }^{(27)}$ or a complementary technique to the above PIXE, such as EPMA for REE and elemental determination, is required. Finally, the EPMA complimentary approach to the PIXE ${ }^{(28)}$ must cover the elementary gaps that the PIXE detects with the $\mathrm{Si}(\mathrm{Li})$ detector but not with the medium energies PIXE.

\section{Conclusions}

The current PIXE approach is well-established for providing useful data for multi-element analysis of complex metamorphic rocks. The proton microprobe outperforms the electron microprobe for both major and trace elements in terms of signal to background ratio. Proton microprobes have some advantages, such as bremsstrahlung. The composition of metamorphic rocks was then quantified utilising an incoming electron beam of $8 \mathrm{keV}$ and $20 \mathrm{nA}$ and the increased capabilities of electron probe micro analyzers.

When comparing EPMA to PIXE analysis, the detection limits for most elements are roughly $100 \mathrm{ppm}$ or times greater. The limit of detection in the case of applying EPMA for current metamorphic rock REE-bearing minerals is normally in the concentration range of 50-500 ppm. However, elements with numerous interference of analytical lines, such as $\mathrm{Gd}, \mathrm{Tm}, \mathrm{Eu}$, and $\mathrm{Lu}$ in REE, have multiple interferences of analytical lines. The detection limit is calculated using the conventional formula used in the case of exact EPMA computations of all interfering lines' overlap coefficients.

The electron and proton microprobe's downside or limitation is measuring the oxidation state of elements like Fe in charnockite material, particularly Fe2+ and Fe3+ content. Previous electron microprobe investigations only reported element oxides, not cations; consequently, cation proportions and mineral formulas must be recalculated using stoichiometry criteria.

The light elements $\mathrm{Li}, \mathrm{Be}, \mathrm{F}, \mathrm{Na}, \mathrm{Al}, \mathrm{Mg}$, Si, and $\mathrm{P}$ due to $\mathrm{K}-\mathrm{X}$-ray energies of less than $2 \mathrm{keV}$ according to $\mathrm{Si}$ (Li) detector dimensions are once again explored in the PIXE with $3 \mathrm{MeV}$ investigations. The light elements, such as lithium, beryllium, and boron, can only be measured using EPMA, with the exception of $Z<6$ from the aforementioned results.

However, prior investigations have shown that EPMA is accurate when detecting high $\mathrm{Z}$ elements like as $\mathrm{Y}, \mathrm{U}, \mathrm{Th}$, and $\mathrm{Pb}$, and REE permits the connection of microstructure and geochronology data, providing geologists with a new type of information. However, due to their high cross-section values, $3 \mathrm{MeV}$ protons do not excite $\mathrm{K}-\mathrm{X}$ rays of high $\mathrm{Z}$ elements in PIXE, and L-X-rays are an issue due to the overlap of $\mathrm{KX}$ rays of light elements.

Parts per million values are enabled by lower bremsstrahlung in proton microprobes; higher ppm values than complementary approaches like EPMA are derived from earlier literature. Due to the effective detection of the KX-ray range Z=20 to $50, \mathrm{~L} \mathrm{X-ray}$ range for $Z>50$, and $Z=20$ to 50 for the $M X$-ray range, low and medium $Z$ elemental concentrations are measured through $K$ $\mathrm{X}$-rays and high $\mathrm{Z}$ elemental concentrations are measured through $\mathrm{L} \mathrm{X}$-rays in the above PIXE analysis of metamorphic rock samples.

Because of its excellent spatial resolution and low detection limits, PIXE has shown to be a valuable technique for characterisation of minerals with complicated chemistry in metamorphic rock samples. At low and high Z, REE that are not detected by PIXE, a proton microprobe is an excellent companion to an electron microprobe.

\section{Acknowledgment}

AVSS expresses his deep gratitude to Asst. Prof. P. V. Laxminarayana, Head of the Department and staff, Department of Nuclear Physics, Andhra University, Visakhapatnam. Also thankful to Prof. Y. Rama Krishna, Head of the Department, Engineering Physics, Andhra University, for the facilities extended to carry out the research work.

\section{References}

1) Sriramadas A, Rao AT. Charnockites of Visakhapatnam, Andhra Pradesh. Journal of Geological Society of India. 1979;20(10):512-517. Available from: http://www.geosocindia.org/index.php/jgsi/article/view/64626.

2) Bhattacharya S. Eastern Ghats granulites terrain of India: an overview. Journal of Southeast Asian Earth Sciences. 1996;14(3-4):165-174. doi:10.1016/s07439547(96)00055-4. 
3) Pownceby MI, MacRae CM, Wilson NC. Mineral characterisation by EPMA mapping. Minerals Engineering. 2007;20(5):444-445. Available from: https://doi.org/10.1016/j.mineng.2006.10.014.

4) Ishii K. PIXE and Its Applications to Elemental Analysis. Quantum Beam Science. 2019;3(2):12-12. doi:10.3390/qubs3020012.

5) Borghi A, Cossio R, Olmi F, Ruffini R, Vaggelli G. EPMA Major and Trace Element Analysis in Garnet and its Petrological Application. Microchimica Acta. 2002;139(1-4):17-25. doi:10.1007/s006040200034.

6) Christopher MH, John LC, Gerald KC. Refinement of major- and minor-element PIXE analysis of rocks and minerals. Nuclear Instruments and Methods in Physics Research B; Beam Interactions with Materials and Atoms. 2016;366:40-50. doi:10.1016/j.nimb.2015.10.018.

7) Ishii K, Yamazaki H, Matsuyama S, Galster W, Satoh T, Budnar M. Contribution of atomic bremsstrahlung in PIXE spectra and screening effect in atomic bremsstrahlung. X-ray Spectrometry. 34(4), pp. 363-365. . X-ray Spectrometry. 2005;34(4):363-365. Available from: https://doi.org/10.1002/xrs.838.

8) Avupati VSS, Rao MJ, Mouli KC, Seetaramireddy B. The Performance of PIXE Technique through a Geochemical Analysis of High Grade Rocks. Journal of Nuclear Physics Material Science Radiation and Applications. 2019;7(1):13-28. doi:10.15415/jnp.2019.71002.

9) Satyanarayana AVS, Rao MJ, Mounika KSS. Need of Complementary Analytical Technique at PIXE-Complex Matrix Composition Analysis. International Journal of Scientific Research in Physics and Applied Sciences. 2020;8(3):36-40. doi:10.26438/ijsrpas/v8i3.3640.

10) Kamineni DC, Bonardi M, Rao AT. Halogen-bearing minerals from Airport Hill, Visakhapatnam, India. American Mineralogist. 1982;67(9-10):1001-1004. Available from: https://pubs.geoscienceworld.org/msa/ammin/article-abstract/67/9-10/1001/41490/Halogen-bearing-minerals-from-Airport-Hill.

11) Chinnasamy PSS, Hazarika D, Pal R, Sen G, Govindaraj. Pyrite Textures and Trace Element Compositions from the Granodiorite-Hosted Gold Deposit at Jonnagiri, Eastern Dharwar Craton, India: Implications for Gold Mineralization Processes. Economic Geology. 2021;116(3):559-579. doi:10.5382/econgeo.4787.

12) Colby JW. MAGIC V-A computer program for quantitative electron excited energy dispersive analysis. Instruction manual QUANTEX RAY, Appendix E, ED-1: 1/8/80, Kevex Corporation, Foster City, California.. 1980.

13) Schulz B, Sandmann D, Gilbricht S. SEM-Based Automated Mineralogy and Its Application in Geo- and Material Sciences. Minerals. 2020;10:1004-1004. Available from: https://doi.org/10.3390/min10111004. doi:10.3390/min10111004.

14) Utui RJ, Wang X, Guerreiro FM, Elfman M, Kristiansson P, Malmqvist KG, et al. Application of nuclear microprobe in the study of granulite facies rocks from the Namama thrust belt, Nuclear Instruments and Methods in Physics Research B; Beam Interactions with Materials and Atoms. Nuclear Microprobe Technology and Applications. 1997;130(1-4):687-69Q. Available from: https://doi.org/10.1016/S0168-583X(97)00300-5.

15) Satyanarayana AVS, Kumar SR, Sharma S. An application of PIXE technique to Proto Crustal Rocks: Geo chemical evaluation of Granulitic Charnockites of Eastern Ghats, Andhra Pradesh. Journal of Nuclear Physics Material Science Radiation and Applications. 2016;3(2):147-155. doi:10.15415/jnp.2016.32016.

16) Shariff MA, Bülow K, Elfman M, Kristiansson P, Malmqvist KG, Pallon J. Calibration of a new chamber using GUPIX software package for PIXE analysis. Nuclear Instruments and Methods in Physics Research Section B; Beam Interactions with Materials and Atoms. 2002;189:131-137. doi:10.1016/S0168583X(01)01018-7.

17) Maxwell JA, Teesdale WJ, Campbell JL. The Guelph PIXE software package II. Nuclear Instruments and Methods in Physics Research B; Beam Interactions with Materials and Atoms. 1995;95(3):407-421. Available from: https://doi.org/10.1016/0168-583X(94)00540-0.

18) Laputina IP, Vadim A, Batyrev AI, Yakushev. A new EPMA technique for determination of rare earth elements with the use of automated peak-overlap and modeled background corrections. Journal of Analytical Atomic Spectroscopy. 1999;3:465-469. doi:10.1039/A806828C.

19) Kabir MH. Particle Induced X-ray Emission (PIXE) Setup and Quantitative Elemental Analysis. 2007. Available from: http://hdl.handle.net/10173/366.

20) Popelka-Filcoff RS. Proton-Induced X-Ray Emission Spectroscopy (PIXE): Applications in Archaeology. In: C S, editor. Encyclopedia of Global Archaeology. Cham. Springer. 2018. Available from: https://doi.org/10.1007/978-3-319-51726-1_342-2.

21) Venkata A, Satyanarayana S, Rao MJ, Reddy BS. Low Z Elements of High Grade Metamorphic Rocks by PIXE Analysis - A Comprehensive Review. Geoscientific Instrumentation Methods and Data Systems. 2020. Available from: https://doi.org/10.5194/gi-2020-40. doi:10.5194/gi-2020-40.

22) Miranda J, Nuclear Instruments and Methods in Physics Research B; Beam Interactions with Materials and Atoms. Low energy PIXE: advantages, drawbacks, and applications. 1996;118(1-4):346-351. Available from: https://doi.org/10.1016/0168-583X(95)01176-5.

23) Hajivaliei M, Puri S, Garg MLL, Mehta D, Kumar A, Chamoli SKK, et al. K and L X-ray production cross sections and intensity ratios of rare-earth elements for proton impact in the energy range 20-25 MeV. Nuclear Instruments and Methods in Physics Research Section B: Beam Interactions with Materials and Atoms. 2000;160(2):203-215. doi:10.1016/s0168-583x(99)00587-x.

24) Satyanarayana A, Rao MJ, Mouli KC. Limitation of uranium and thorium traces in charnockite matrix-PIXE analyses at $3 \mathrm{MeV}$ with $\mathrm{Si}(\mathrm{Li})$ detector. Journal of Radioanalytical and Nuclear Chemistry. 2021;328:83-87. doi:10.1007/s10967-021-07623-0.

25) Satayanarayana AVS, Rao MJ, Mouli KC, Reddy BS. Review on PIXE for Detection of Rare Earth Elements in Charnockite Complex Matrix Composition, Journal of Radiation and Nuclear Applications. 5(3), pp. 175-180 . Journal of Radiation and Nuclear Applications. 2020;5(3):175-180. Available from: http://dx.doi.org/10.18576/jrna/050302.

26) Satyanarayana AVS, Rao MJ. Various Experimental Factors Behind the Missing Elements in PIXE Spectrum of Charnockite Matrix. Journal of Nuclear Physics Material Science Radiation and Applications. 2020;8(1):33-42. doi:10.15415/jnp.2020.81005.

27) Venkata A, Satyanarayana S, Rao MJ, Reddy BS. Requirement of HE-PIXE at High Z Elements in Charnockite Matrix Composition. Annales Geophysicae. 2020. doi:10.5194/angeo-2020-77.

28) Dai Z, Ren C, Zhang J, Ma G, Yang F. Comparison of quantitative PIXE and EPMA microanalysis of mineral samples. Nuclear Instruments and Methods in Physics Research Section B: Beam Interactions with Materials and Atoms. 1995;104(1-4):489-493. doi:10.1016/0168-583x(95)00473-4. 